\title{
Asteroid observations with the Hubble Space Telescope ${ }^{\star}$ FGS $^{\star \star}$
}

\section{Duplicity search and size measurements for 6 asteroids}

\author{
P. Tanga ${ }^{1,3}$, D. Hestroffer ${ }^{2, \star \star \star}$, A. Cellino ${ }^{3}$, M. Lattanzi ${ }^{3}$, M. Di Martino ${ }^{3}$, and V. Zappalà ${ }^{3}$ \\ 1 Laboratoire Cassini, Observatoire de la Côte d'Azur, BP 4229, 06304 Nice, France \\ 2 IMCCE, UMR CNRS 8028, Paris Observatory, 77 Av. Denfert Rochereau 75014 Paris, France \\ 3 INAF, Osservatorio Astronomico di Torino, Strada Osservatorio 20, 10025 Pino Torinese (TO), Italy
}

Received 19 August 2002 / Accepted 9 December 2002

\begin{abstract}
We present the results of the observations of five Main Belt asteroids and one Trojan obtained using the Fine Guidance Sensors (FGS) of the Hubble Space Telescope. For each object, estimates of the spin axis orientation, angular size and overall shape, as well as possible indications of a binary structure, are derived. This enables the computation of new physical ephemerides. While the data concerning (63) Ausonia are clearly compatible with a three-axis ellipsoidal model, other objects show more complex shapes. (15) Eunomia, (43) Ariadne and (44) Nysa could in fact be double asteroids, or highly irregular bodies. The data concerning (624) Hektor are not conclusive as to its supposed binary nature, even if they agree with the signal of a single body. The results presented here strongly support the outstanding capabilities of the FGS for asteroid measurements, provided that the observations are performed over a sufficient time interval.
\end{abstract}

Key words. minor planets, asteroids - methods: observational

\section{Introduction}

The HST/FGS astrometer has already been successfully used in the past to derive angular diameters and flattenings of Mira stars (Lattanzi et al. 1997) with typical sensitivities around the $\sim 1$ mas level. An application of FGS to the measurement of Solar System bodies (minor planets in particular) has been suggested in the past, and the results presented here are the first successful attempts that prove that the HST/FGS is valuable in measuring the apparent angular sizes of minor bodies and in providing important information on their shapes. Five main-belt asteroids and one Jupiter Trojan have been observed as part of an approved program devoted to search for duplicity among asteroids during HST Cycle 8 (Zappalà et al. 1998; Hestroffer et al. 2002a). The targets ((15) Eunomia (43) Ariadne; (44) Nysa, (63) Ausonia, (216) Kleopatra and (624) Hektor) were selected on the basis of peculiar photometric properties suggesting a possible binarity (see Leone et al. 1984; Cellino et al. 1985). Apart from

Send offprint requests to: $\mathrm{P}$. Tanga,

e-mail: tanga@obs-nice.fr

* Based on observations with the NASA/ESA Hubble Space Telescope, obtained at the Space Telescope Science Institute, which is operated by the Association of Universities for Research in Astronomy, Inc. under contract No. NAS5-26555.

$\star \star$ Figures 1 to 12 are only available in electronic form at http://www . edpsciences.org

$\star \star \star$ Invited researcher at the Osservatorio Astronomico di Torino. their large maximum amplitudes, the light-curves of the selected targets are generally compatible with the behavior expected for couples of bodies having overall rubble pile internal structures, for which equilibrium shapes can be expected (Weidenschilling 1980; Zappalà et al. 1983). In particular, the observed rotation periods and light-curves could be consistent with contact - or nearly-contact - binaries that could form as outcomes of catastrophic collisions with large angular momentum transfer. Of course, this is not the only possible interpretation of the available data, since purely shape effects can be responsible for light-curve morphologies like those observed for these objects (Cellino et al. 1989). As shown in a previous paper (Hestroffer et al. 2002b, called hereafter Paper I), the HST/FGS instrument is powerful enough to resolve such binary systems or to find constraints for alternative shape models.

Our observations show that there is no compelling evidence for well separated or nearly-contact binaries among the objects of our sample. The HST/FGS nevertheless provides important and accurate results on the pole orientation, size, shape, and brightness distribution of these asteroids, hence enabling the computation of a physical ephemeris. The goodness of fit obtained assuming a single triaxial ellipsoid shape varies among the different objects of our sample. While (44) Nysa and (63) Ausonia are very well modeled by prolate spheroids, the data obtained for (15) Eunomia, (43) Ariadne and (624) Hektor show slight but appreciable departures from this ideal shape. (216) Kleopatra is confirmed 
to have a bi-lobated dumbbell shape (see also Tanga et al. 2001) as suggested by previous observations (Ostro et al. 2000; Marchis et al. 1999).

A methodological discussion on the basic strategy was presented in Paper I, together with the principles employed in the data reduction. In this second paper we present the detailed results obtained for each of the observed bodies. In Sect. 2 the observing circumstances are given. The results are developed in Sect. 3, together with the physical parameters (pole orientation, shape and size estimate) that have been derived.

\section{Observations and data reduction}

\subsection{Strategy}

The ephemerides of the targets at the epochs of their observations and the observing logs are given in Table 1 . The observations were carried out using the FGS astrometer (FGS\#3 for all objects, with the exception of (216) Kleopatra, observed by FGSR\#1). In order to improve the signal-to-noise $(S / N)$ ratio, the multiple scan merging strategy used by Lattanzi et al. (1997) was followed. Four to six consecutive scans were made before each re-centering; this set of target acquisition and observation is called a "visit". Each object was observed during a whole HST “orbit”, i.e. a sequence of visits distributed all along a continuous visibility period of the asteroid as seen from HST. The position angle of the FGS axes did not change during the orbit. The FGS was used with a step delay of $0.025 \mathrm{~s}$. Each sampling step was 1.0 or 1.5 mas, with a total scan duration of 160 or $120 \mathrm{~s}$, respectively. Only in the case of (624) Hektor, was the sampling step 2.4 mas, and the total scan duration $150 \mathrm{~s}$. In all cases, these parameters correspond to a total scan length of 2 arcsec. All observations were performed with the PUPIL filter which - although penalizing in limiting magnitude - reduce the negative effect of the spherical aberration of HST optics.

Due to the limited time-allocation, only a relatively small fraction $(5-10 \%)$ of the total spin period, corresponding to the duration of each orbit, is covered by the observations. Since the main purpose of this program was the detection of nearlycontact binary systems, the asteroids were observed near their predicted light-curve maximum, approximately corresponding to the maximal apparent separation of possible components. The observations of each object were carried out at an epoch corresponding to a given value of the aspect angle $\xi$. As a consequence, the complete three-dimensional shape of the asteroids cannot be completely and unambiguously retrieved. In particular, assuming for the sake of simplicity that an object is a perfect triaxial ellipsoid with axes $a>b>c$ observed at the epoch of the maximum light-curve, one should expect that the projected area on the sky will be an ellipse, having axes equal to $a$ (longest apparent axis) and $\sqrt{b^{2} \cos ^{2} \xi+c^{2} \cos ^{2} \xi}$ (shortest axis), respectively. According to the value of the aspect angle $\xi$, the relative contribution of the semiaxes $b$ and $c$ to the corresponding shortest axis of the projected ellipse varies significantly, although one could expect a priori that a pole-on view (leading to full determination of $b$ and complete indetermination of $c$ ) is rarely achievable in practice, whereas an aspect closer to equatorial view (corresponding to full determination of $c$ and complete indetermination of $b$ ) is more likely. In principle, having the possibility to follow an object during a significant fraction of its rotation period would lead to a much better determination of the overall shape. In our observations, we have tried to maximize the information coming from the slow, steady change of the apparent ellipse projected by each object, although it is clear that a longer available observation time would have allowed us to carry out a much better reconstruction of the true three-dimensional shapes.

The recorded signal is an "S-shaped" curve (usually called an "S-curve") whose detailed shape depends upon the target size, shape, and surface brightness distribution. As explained in detail in Paper I, the "S-curve" allows us to distinguish a close binary object, such as those searched for by this observing program. Many examples are shown in this paper (Figs. 7-14) and discussed in the text in the following Sections.

\subsection{Data reduction}

As explained in Paper I, the data over successive scans (corresponding to less than $5 \mathrm{mn}$ of time) are merged to obtain a higher $S / N$ ratio. For (624) Hektor, a smoothing of data by low-pass filtering was performed before merging. A single $\mathrm{S}$ curve is thus obtained for each FGS axis and each visit. Because the targets were at a few AU distance, the data were corrected for the apparent motion of the target during the scan, produced by the displacement of the HST platform along its orbit ${ }^{1}$. This correction corresponds to a re-scaling of the FGS-axis abscissa. Synthetic S-curves are subsequently calculated by convolution of a template transfer function with a shape model, taking into account a specific brightness distribution. Different template files acquired in 1998 and 1999 have been made available by the STScI. They were acquired in 1998 and 1999 and correspond to stars Upgren $69(B-V=0.5$, file $f 44 \mathrm{v} 0702 \mathrm{~m}$ in the calibration database) and HD $233877(B-V=1.1$, file $\mathrm{f} 43 \mathrm{p} 0501 \mathrm{~m})$, whose color indexes are close to that of a typical asteroid. No use is made of piecewise interpolated data, but the value of $T(x)$ for any abscissa $x$ is obtained by linear interpolation. Except for the asteroid (216) Kleopatra, the calibration data obtained in 1998 for the HD 233877 star was used for the transfer function.

Concerning single body shape models, only perfect triaxial ellipsoid shapes have been considered in this analysis. On one hand, this is justified by the purpose of finding basic information on the overall shapes of the objects, avoiding as a first step excessive and unnecessary complexity; on the other hand, the limited coverage of the rotational phase and the single aspect angle covered by the observations do not permit us to analyze much more complex models. As we will see, simple shapes are sufficient, in general, to reproduce the overall features of the observations, and to identify interesting discrepancies when present. Finally, for what concerns possible binarity,

\footnotetext{
1 The displacement due to the orbital motion of the targets themselves during each scan were negligible.
} 
Table 1. Selected targets ephemeris and observation logs.

\begin{tabular}{|c|c|c|c|c|c|c|c|c|c|c|c|}
\hline \multirow[b]{2}{*}{ Name } & \multirow[b]{2}{*}{ Date } & \multicolumn{2}{|c|}{ UTC time of visit } & \multirow{2}{*}{$\begin{array}{c}\text { Mag. } \\
V\end{array}$} & \multirow{2}{*}{$\begin{array}{c}\text { Sidereal } \\
\text { period }^{(b)} \\
{[\mathrm{h}]}\end{array}$} & \multirow{2}{*}{$\begin{array}{c}\text { Geoc. } \\
\text { Dist. } \\
{[\mathrm{AU}]}\end{array}$} & \multirow{2}{*}{$\begin{array}{c}\text { Solar } \\
\text { phase } \\
{[\mathrm{deg}]}\end{array}$} & \multirow{2}{*}{$\begin{array}{l}\operatorname{SEP}^{(a)} \\
\lambda \quad \beta \\
{[\mathrm{deg}]}\end{array}$} & \multicolumn{3}{|c|}{ Scans } \\
\hline & & $\begin{array}{c}\text { first } \\
{[\mathrm{h}]}\end{array}$ & $\begin{array}{c}\text { last } \\
{[\mathrm{h}]}\end{array}$ & & & & & & $\begin{array}{c}\text { step } \\
\text { [mas] }\end{array}$ & $\#$ & $\begin{array}{c}\text { roll }^{(\mathrm{c})} \\
{[\mathrm{deg}]}\end{array}$ \\
\hline (15) Eunomia ${ }^{(\mathrm{d})}$ & 30 Sep. 98 & $23: 30: 03$ & $00: 05: 10$ & 8.6 & 6.0828 & 1.360 & 20.7 & $126-8.4$ & 1.5 & 4 & 42.1 \\
\hline (43) Ariadne & 22 Aug. 98 & $17: 14: 45$ & $17: 51: 47$ & 10.3 & 5.7620 & 0.958 & 18.6 & $145+44.4$ & 1.0 & 4 & 273.0 \\
\hline (44) Nysa & 05 Sep. 98 & 18:40:09 & 19:17:11 & 10.6 & 6.4214 & 1.638 & 17.2 & $221-6.5$ & 1.0 & 4 & 52.9 \\
\hline (63) Ausonia & 02 Apr. 98 & 16:07:08 & $16: 40: 32$ & 11.7 & 9.2976 & 1.824 & 19.7 & $304+57.1$ & 1.0 & 4 & 287.6 \\
\hline (216) Kleopatra & 13 Jan. 00 & $13: 32: 59$ & $14: 11: 17$ & 10.8 & 5.3853 & 1.529 & 22.4 & $57-43.0$ & 1.0 & 2 & 248.7 \\
\hline (624) Hektor & 23 Oct. 98 & $12: 03: 52$ & $12: 42: 43$ & 15.0 & 6.9205 & 4.618 & 9.5 & $313-32.5$ & 2.4 & 6 & 55.1 \\
\hline
\end{tabular}

(a) Coordinates of the Sub-Earth Point (SEP) with respect to the asteroid equator, following the pole coordinates given in Table 2. The longitude origin is assumed to be at a semi-meridian lying on the plane that contains the major axis, and it is given for the first visit.

(b) From P. Magnusson's on-line database: http://www. astro.uu.se/ per

(c) Position angle of the FGS-X axis (also called "roll angle") with respect to the North direction and counted positive toward the East.

(d) Last visit on October 1st.

we assumed that the binary components are triaxial ellipsoids in synchronous rotation, with major axes mutually aligned and parallel spin axes, accordingly with the original idea of possible equilibrium models suggested by available light-curves (Cellino et al. 1985).

\section{Modeling asteroid shapes}

A grid of triaxial ellipsoid models is explored as a first step to obtain a preliminary best fit for each single visit, separately for each FGS-axis. In a second step, a model-grid is explored to obtain the best fit solution for the whole set of visits taking into account the asteroid rotation. This iterative two-steps fitting procedure finally provides the pole solution $(\lambda, \beta)$, the lengths of the axes of the ellipsoid $(a, b, c)$, the separation and the axes $\left(a^{\prime}, b^{\prime}, c^{\prime}\right)$ of the secondary in case of a binary, and the rotational phase angle $W_{0}$, i.e., the position of the major-axis meridian at a given reference epoch (see Hilton 1992; Seidelmann et al. 2002). In all cases, a fit assuming two components with diameter ratio larger than 0.5 and varying separation has been performed to check the possible binary nature. If the goodness of the fit is poor, the hypothesis of a nearly-contact binary system can be rejected. A complete discussion of this procedure can be found in Paper I.

The values obtained for the physical ephemeris and the ellipsoid sizes are summarized in Table 2. They were computed assuming uniform surface brightness of the projected asteroid shape. The error bars of each listed quantity depends critically on the orientation of the asteroid with respect to the direction of the two FGS axis. For this reason, details are given in the discussion concerning each single object. However, in general the formal error (depending upon the asteroid magnitude) is of the order of a few mas for the best determined axis, and can be as large as $10-20 \%$ for the less well constrained length ${ }^{2}$. Moreover, these formal errors refer to the best-fit ellipsoidal

${ }^{2}$ The contribution of the $b$ and $c$ semiaxes to the projected ellipse measured in the sky around the maximum of the light-curve is mainly a function of the aspect angle, as explained above. For instance, in the limiting case of an object observed at a perfectly polar view (aspect model, which may in principle differ from the actual shape of the observed asteroid. Last, as seen in Sect. 2.1, the modeling is based on a limited variation of the apparent projected ellipse during each visit as a consequence of asteroid rotation. In this respect, faster rotations can provide in principle a better reconstructed shape.

Our data analysis procedure yields the best-fit solution for a triaxial ellipsoid assuming a uniform brightness distribution. Introducing a limb-darkening effect of the surface leads to larger resulting sizes with approximately the same goodness of fit. The exact function describing this limb-darkening is generally not known for the asteroids and cannot be retrieved from our data alone. However, in order to quantify the uncertainty in the resulting shape and size determination due to the insufficient knowledge of limb-darkening, we have performed a separate data reduction by assuming a normalized brightness distribution corresponding to a Minnaert's law (Minnaert 1941), $I=\mu_{0}^{k} \mu^{k-1}\left(\mu_{0}\right.$ and $\mu$ being the cosine of the incidence and reflection angles, respectively) assuming $k=0.6$ (Hestroffer 1998; Parker et al. 2002), i.e., moderate limb-darkening. We found that, except for (15) Eunomia, the systematic error on size estimate is of the order of $3 \%$. Interestingly, we also found that introducing a limb-darkening effect has no appreciable influence on the resulting ellipsoid flattening. In other words, while the overall size increases slightly for increasing limbdarkening, the change on the resulting $a / b$ and $a / c$ ratios tends to be insignificant.

In the following sections we give, for each asteroid, the results for the duplicity test and the data inversion. The axial ratios of the ellipsoids are compared to the values derived by Magnusson et al. (1994) (and reference therein) on the basis of photometric analyses. The same source provides a set of pole coordinates for each object, as determined by different authors. Coordinate values are normally spread over two intervals of a few degrees, grouped around two independent pole orientations, both compatible with the available photometric data (Taylor 1979). The two orientations differ by about

angle $\xi=0^{\circ}$ ), no indication of the length of the smallest axis $c$ would be possible for any possible rotational phase. 
Table 2. Physical ephemeris and shape parameters derived from HST/FGS observations. For (216) Kleopatra the solution with two overlapping ellipsoidal components is given.

\begin{tabular}{|c|c|c|c|c|c|c|c|c|c|}
\hline \multirow[t]{2}{*}{ Name } & \multicolumn{5}{|c|}{ Pole $^{(a)}$ and rotation ${ }^{(\mathrm{b})}$} & \multicolumn{4}{|c|}{ Ellipsoid $^{(\mathfrak{c})}$} \\
\hline & $\lambda$ & $\begin{array}{r}\beta \\
{[\mathrm{deg}]}\end{array}$ & $W_{0}$ & $\begin{array}{r}\text { Ref. ep } \\
\text { [h UT }\end{array}$ & & $\begin{array}{lll}a & b & c \\
& {[\mathrm{mas}]}\end{array}$ & $\begin{array}{lll}a & b & c \\
& {[\mathrm{~km}]}\end{array}$ & $a / b$ & $a / c$ \\
\hline (15) Eunomia & 352 & -58 & 34.8 & $01 / 09 / 82$ & 09.1 & 183104103 & 181103102 & $(1.76)$ & 1.78 \\
\hline (43) Ariadne & 252 & -16 & 111.0 & $08 / 16 / 85$ & 03.3 & $\begin{array}{lll}65 & 38 & 38\end{array}$ & $\begin{array}{lll}45 & 26 & 26\end{array}$ & 1.71 & $(1.71)$ \\
\hline (44) Nysa & 102 & +50 & 254.8 & $09 / 10 / 79$ & 16.0 & $\begin{array}{lll}50 & 31 & 31\end{array}$ & $\begin{array}{lll}59 & 37 & 37\end{array}$ & $(1.61)$ & 1.61 \\
\hline (63) Ausonia & 119 & -29 & 15.3 & $01 / 23 / 83$ & 23.5 & $\begin{array}{lll}57 & 25 & 25\end{array}$ & $\begin{array}{lll}76 & 33 & 33\end{array}$ & 2.28 & $(2.28)$ \\
\hline (216) Kleopatra & 72 & +16 & 248.4 & $05 / 08 / 84$ & 11.8 & & (see Table 3) & & \\
\hline (624) Hektor & 329 & -25 & 63.9 & $02 / 04 / 65$ & 04.5 & $\begin{array}{lll}62 & 28 & 28\end{array}$ & $208 \quad 94 \quad 94$ & 2.21 & $(2.21)$ \\
\hline
\end{tabular}

(a) Solution number of Magnusson et al. (1994) and coordinates in the ecliptic B1950.

(b) The rotational phase $W_{0}$ is computed for the reference epoch given on the second line.

(c) The ellipsoid's flattening coefficient given in parenthesis is determined with lower precision (see text).

Table 3. Same as Table 2 for (216) Kleopatra. Here the solution with two "overlapping" ellipsoidal components is given.

\begin{tabular}{|c|c|c|c|c|c|c|c|}
\hline \multicolumn{3}{|c|}{ Primary } & \multicolumn{3}{|c|}{ Secondary } & \multirow{2}{*}{\multicolumn{2}{|c|}{$\frac{\text { Separation }}{\mathrm{d}}$}} \\
\hline$a b$ & $c$ & $a / b \quad a / c$ & $a^{\prime}$ & $c^{\prime}$ & $a^{\prime} / b^{\prime} \quad a^{\prime} / c^{\prime}$ & & \\
\hline [mas] & {$[\mathrm{km}]$} & & [mas] & {$[\mathrm{km}]$} & & [mas] & {$[\mathrm{km}]$} \\
\hline 693416 & 763818 & 2.03 & 653123 & 723426 & $2.10(2.83)$ & 114 & 125 \\
\hline
\end{tabular}

180 degrees in ecliptic longitude. This is due to the fact that, for both orientations, the same fraction of the asteroid surface is visible from Earth at a given epoch; as a consequence, the integrated disk photometry is the same. However, the orientation of the projected shape of the object on the sky plane is different, therefore high-resolution observations can discriminate between the two solutions. In general, as it will be seen in the detailed discussion about each object, a single pole orientation is consistent with the HST/FGS data. Thus, we are able to eliminate the residual pole ambiguity.

On the other hand, we are not able to provide fully independent improvements of the precise value of pole coordinates, that would require observations over longer time spans, at different aspect angles. Thus, we did not include the pole coordinates in the free parameters of the fit. In fact, our observations (being restricted to a single aspect angle) are mainly sensitive to the projected position of the rotation axis on the sky plane, not its real position in space. Given the large uncertainty (several degrees) affecting the pole coordinates given in Magnusson et al. (1994), we ran several fit solutions, trying to minimize the residuals, for different values of pole coordinates. The values retained for the final fit, listed in Table 2, are always inside the uncertainty interval of the available solutions. Finally, we should note that the different photometrically-derived pole solutions available in the literature are also associated with some corresponding estimates of the axial ratios $b / a$ and $c / a$, computed from the analysis of the light-curve properties under the general assumption that the objects are triaxial ellipsoids. Our HST observations are able to determine the axial ratios of the objects, given the measured (varying) axial ratios of the projected ellipses in the sky, and assuming different pole solutions. This allows us also to discriminate among different pole solutions in some cases like (43) Ariadne (see below), in which the resulting axial ratios corresponding to one of the pole solutions would be unrealistic (extreme flattening) and totally not compatible with the values derived from the photometry.

A comparison to the asteroid sizes derived by indirect methods, such as radiometric diameters computed on the basis of IRAS-measured thermal IR fluxes, requires one to translate the derived shape parameters into average radii. In the following we discuss our results by computing the radius of a sphere equivalent in volume $\left(R_{\mathrm{v}}\right)$. For a three-axis ellipsoid, we thus have $4 / 3 \pi a b c=4 / 3 \pi R_{\mathrm{v}}^{3}$.

The data for the diameters comparison are summarized in Table 4. The graphs for the final step of our model fit process - providing the ellipsoid parameters - are given in Figs. 1 to 6. The fits of the derived model to the data ("first step") follow (Figs. 7 to 12).

\section{(15) Eunomia}

With an apparent size of approximately 0.26 arcsec, (15) Eunomia is the largest asteroid observed within this program, and hence exhibits the flattest S-curve. The evolution of the S-curve as a function of time (Fig. 1) clearly shows that the size along the $X$-axis remains approximately constant while the size along the other axis is increasing. This enables us to discriminate between the two possible pole solutions. The one that is retained shows that, consistently with the variation observed along the FGS axis, at the epoch of observation (15) Eunomia was almost equator-on with a sub-earth point (SEP in the following) longitude close to $120^{\circ}$. In this viewing conditions, the 
length of the longest and shortest axes ( $a$ and $c$ ) are well determined, whereas the length of the $b$ axis is poorly constrained.

The fit with a single-body solution and the pole solution indicated in Table 2 is acceptable for the whole set of visits (see Fig. 1). (15) Eunomia is an almost prolate-spheroid with sizes $361 \times 205 \times 203 \mathrm{~km}$. The orientation relatively to the FGS axis is such that the lengths of the $a$ and $c$ axes are well determined. The ellipsoid flattening is larger than the one derived from the photometry $(a / b=1.42, a / c=1.6)$ even if $b$ is not well constrained. The derived volume corresponds to an effective diameter of $248 \mathrm{~km}$, close to the IRAS diameter $(255 \mathrm{~km})$.

In contrast with the other asteroids of this program, the large size of (15) Eunomia, combined with the relatively large solar phase angle, implies that introducing a moderate limbdarkening (Minnaert $k \sim 0.6$ ) provides a slightly better goodness of fit to the data.

A more careful analysis of fit residuals provides further insight into the the shape of this object. In fact, it can be seen that the S-curves in the $X$-axis are particularly asymmetric in comparison to the model. The fit residual, systematically present especially close to the S-curve maximum (Fig. 7), can reflect a shape or brightness-distribution irregularity (i.e. presence of a spot, non convex or non-symmetric shape, etc.). To test this hypothesis, a dark-spot - as was suggested by Lupishko et al. (1984) on basis of photometric data analysis - was introduced in the fitting grid with varying position, relative albedo, and diameter. The residuals - on a single-scan basis - improved considerably on the $X$-axis, without affecting the fit on the $Y$-axis. Nevertheless, the best-fit dark-spot is much too large (about $25 \%$ of the visible surface) and/or too dark to be realistic. Such model would thus be in complete disagreement with the observed photometric light-curves of this asteroid.

Such a solution being now discarded, a tentative fit with a binary structure and with diameters ratio (secondary/primary) smaller than 0.6 has been done. The residuals of each independent single visit are, again, considerably improved on the $X$-axis. Nevertheless, no acceptable solution fitting together all data on both $X$ and $Y$ axis, can be found. In conclusion, the available data show that duplicity is not convincingly suggested by the data at our disposal.

It should be stressed that this example clearly illustrates the need to have more than two single baselines for the interferometer, and that observations with a different scanning geometry (i.e. different "roll" position angle) would be valuable for the shape reconstruction. As shown in Paper I, an egg-shaped convex profile (Gaffey \& Ostro 1987) or an octants-shape model (Cellino et al. 1989) would provide features similar to those observed on the present S-curves (see Fig. 13). In summary, (15) Eunomia is hardly a binary system nor a regular ellipsoid, but this work confirms that probably it has an egg-like shape that could be accurately modeled with more HST/FGS data.

\section{(43) Ariadne}

In contrast to the other asteroids of this program, two different published poles yield a possible solution for the triaxial ellipsoid that adequately fit the data. Nevertheless, the shape
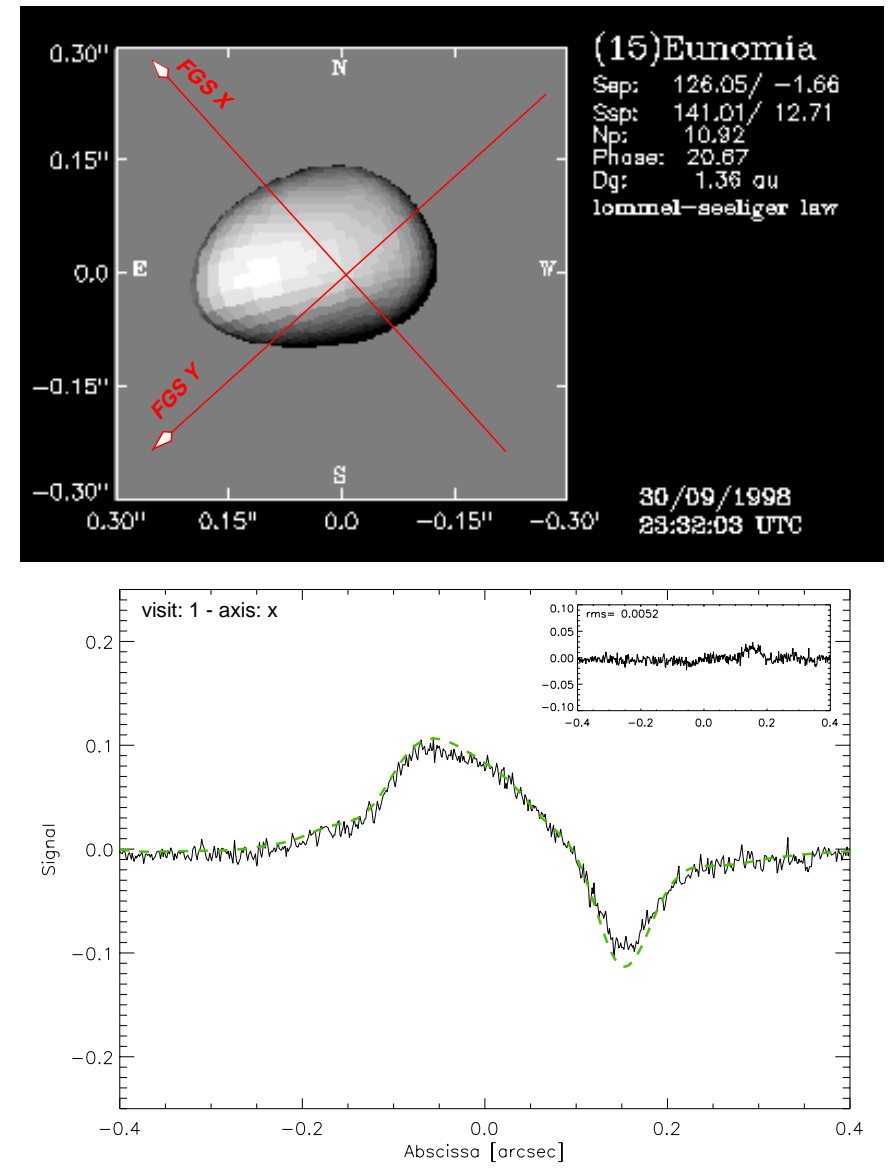

Fig. 13. Octants shape model for (15) Eunomia on the first visit (top), and interferogram (bottom).

corresponding to one of the two solutions would be unrealistic, being characterized by $(a: b: c)=(1: 1: 0.26)$. As we will discuss in more detail in a future work, such a flattened body is not compatible with photometric observations. Our results suggest that (43) Ariadne should be a prolate-spheroid with axial lengths $90 \times 53 \times 53 \mathrm{~km}$. At the epoch of observation, however, (43) Ariadne had an intermediate aspect angle (with SEP latitude $44^{\circ}$ ), so that the length of the (c) axis is not well constrained. The ellipsoid flattening is fairly in agreement with that derived from photometry $(a / b=1.6, a / c=1.8)$ taking into account the uncertainty on $c$. The derived volume corresponds to an effective diameter of $63 \mathrm{~km}$, close to the IRAS diameter $(66 \mathrm{~km})$.

Although the single-ellipsoid model provides an acceptable goodness of fit, the S-curves in the $Y$-axis exhibit a tendency to a slight, increasing deviation from the model at the end of the observing run (see Fig. 8). In fact, a better goodness of fit is obtained with a binary model in contact where the components diameters are in the ratio 0.35 (see Fig. 14). Such a binary model, however, would not adequately reproduce the observed light-curves, and it is not clear from the available data if the components are actually separated or if (43) Ariadne could be a single object with non-ellipsoidal shape. In particular, the actual shape of the "secondary" (a sphere here) is not well constrained. More data are certainly needed to refine the overall shape of (43) Ariadne, but for the moment we have strong 

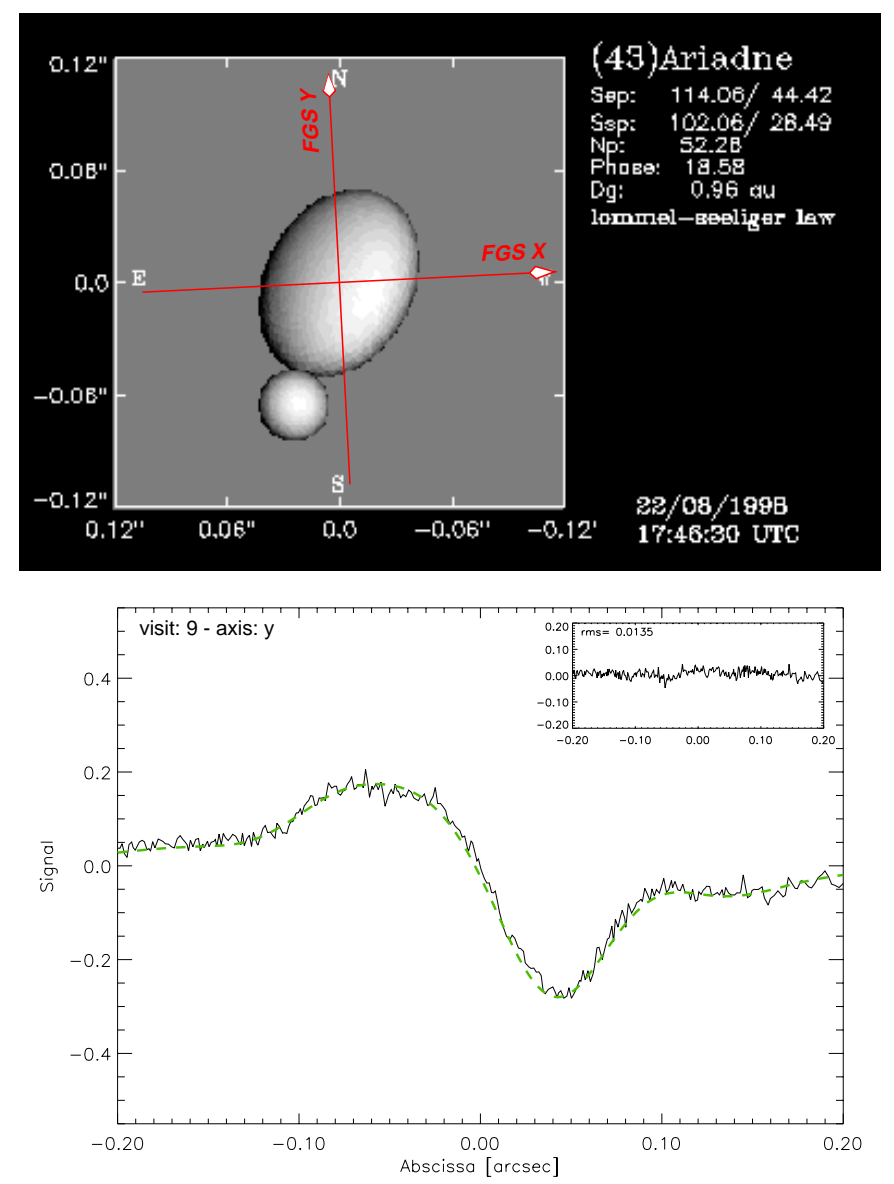

Fig. 14. "Binary" shape model for (43) Ariadne on the last visit (top), and interferogram (bottom).

indication that the asteroid is a bi-lobated or bifurcated nonconvex body. In any case, our data reliably reject the tentative model of Cellino et al. (1985) (based on photometric analysis) that predicts a slightly separated binary system having nearly equal components (diameter ratio 0.93 ).

\section{(44) Nysa}

The fit with a single-body solution and the indicated pole solution is good for the whole set of visits (see Figs. 3 and 9). Any ambiguity on the pole coordinates is thus removed. Nysa is well modeled by a prolate-spheroid of size $119 \times 69 \times 69 \mathrm{~km}$. At the epoch of observation this asteroid was almost equatoron with a SEP longitude close to $220^{\circ}$, so that the major and minor axis $(a$ and $c$ ) are well determined while the intermediate one $b$ ) is poorly constrained. The ellipsoid flattening is coherent with the one derived from the photometry analysis $(a / b=1.44, a / c=1.6-2.3)$ taking into account the inherent uncertainty on $b$. The derived volume corresponds to an effective diameter of $83 \mathrm{~km}$, larger by $16 \%$ than the IRAS diameter $(71 \mathrm{~km})$.

Kaasalainen et al. (2002), from analysis of photometric data, derive a cone-like shape for (44) Nysa, that they suggest to be the signature of a "compound asteroid" consisting of two components of unequal size. Although there is no strong
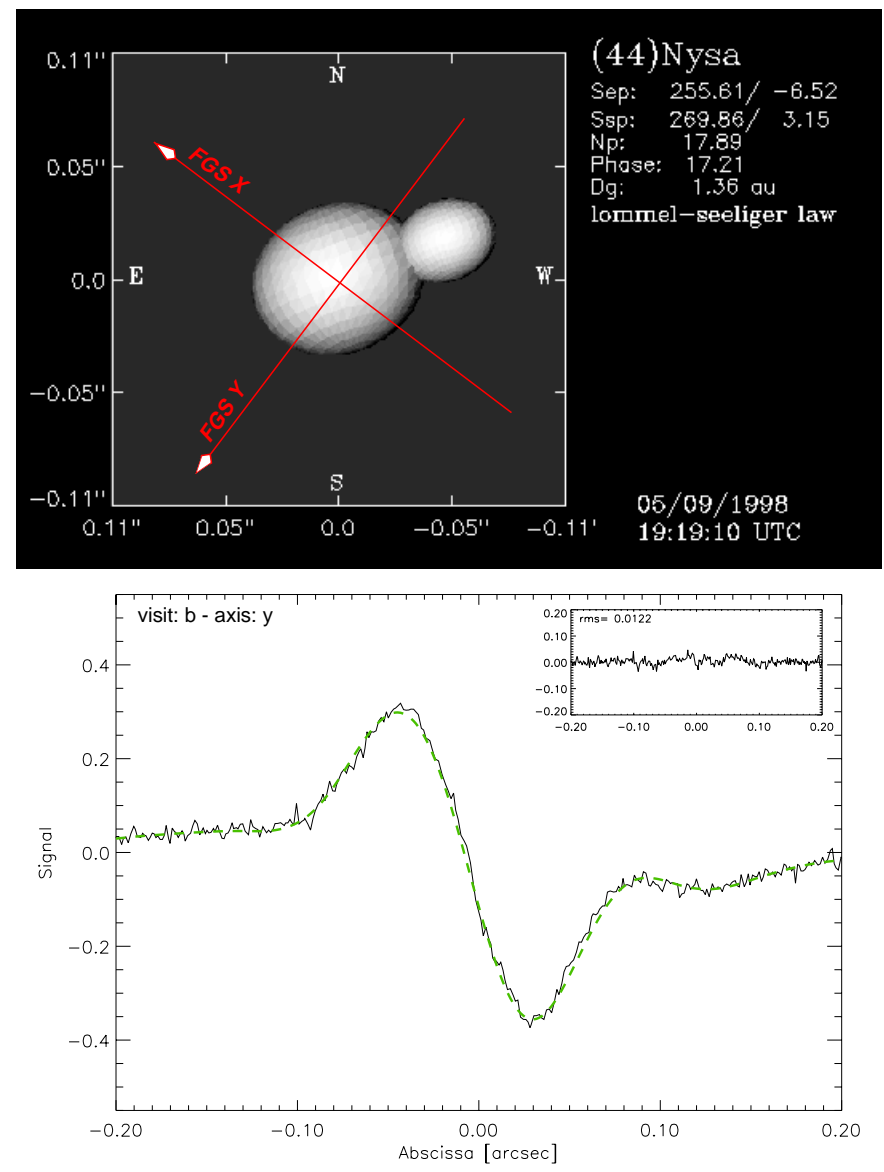

Fig. 15. "Binary" shape model for (44) Nysa on the last visit (top), and interferogram (bottom).

indication of such a contact-binary structure in the available HST/FGS data for this object, we tested this hypothesis by fitting the data with a binary model. The best-fit solution, given in Fig. 15, is obtained for a contact structure with a 0.6 diameter ratio. It is stressed that the goodness of fit is neither considerably improved nor is it degraded in this case, and that hence the FGS data alone cannot confirm or rule out such a solution. A more careful analysis, combining both photometric and interferometric data, should help to constrain a possible non-convex model and reveal the actual shape of (44) Nysa.

\section{(63) Ausonia}

The fit of the observations by means of a single triaxial ellipsoid model is the best we could find in our sample. In fact, residuals are very small for the whole set of visits (see Figs. 4 and 10). (63) Ausonia is not a binary asteroid but a regular prolate spheroid with sizes $151 \times 66 \times 66 \mathrm{~km}$.

At the epoch of observation the SEP latitude for (63) Ausonia was large $\left(57^{\circ}\right)$, so that the length of the smallest axis $(c)$ is poorly constrained. The ellipsoid flattening is in good agreement with the one derived from the photometry analysis $(a / b=2.2, a / c=2.2)$, and in particular with the resulting shape of Zappalà \& Knezevic (1984). The derived volume corresponds to an effective diameter of $87 \mathrm{~km}$, smaller by $16 \%$ than the IRAS diameter $(103 \mathrm{~km})$. 


\section{(216) Kleopatra}

Unlike the other asteroids of our sample, the observed S-curves of (216) Kleopatra are not consistent with a single triaxial ellipsoid model, but are best explained by a double-lobed shape model with the pole indicated in Table 2 (Fig. 5). The fit procedure is based on assuming an ellipsoidal companion with varying size, flattening, and separation. The best-fit model is obtained by using two similarly sized elongated bodies overlapping each other. Consistently to their volume, we call them "primary" and "secondary". The details about this solution are given in (Tanga et al. 2001). This bi-lobated model is coherent with the radar observations of Ostro et al. (2000) and adaptive optics observations of Marchis et al. 1999.

At the epoch of observation the SEP latitude for (216) Kleopatra was large $\left(-43^{\circ}\right)$, so that the length of the smallest axis $(c)$ is poorly constrained. No direct comparison should be done with the ellipsoid flattening derived from photometry. Nevertheless the resulting $a / b$ ratio for the "primary" and the "secondary" is of the order of 2.1, which is still a somewhat high value for such a large asteroid. Due to the peculiar shape, different from the simple models usually employed to derive sizes from thermal data, a comparison with the IRAS diameter can only be tentative. Taking into account that the two ellipsoids of the model are overlapping by approximately $10 \%$, the derived volume corresponds to an effective diameter of $95 \mathrm{~km}$, smaller than the IRAS diameter $(135 \mathrm{~km})$.

The fit of the S-curves - while satisfactory - is not as good as for other asteroids in this program (see Fig. 11), and reflects non-modeled shape and/or brightness anomalies. Nevertheless, it is shown in Hestroffer et al. (2002c) that such a simple model of overlapping ellipsoids better reproduces the presently observed S-curves than would the topographic nominal model obtained by Ostro et al. (2000) from inversion of radar data.

\section{(624) Hektor}

(624) Hektor, a member of the Jupiter Trojans, is the only non main-belt asteroid in this program. It is also the faintest object observed, close to the limit of acceptable $S / N$ ratio for the FGS\#3. At the epoch of observation the best-fitting pole solution suggested a SEP latitude relatively large $\left(-33^{\circ}\right)$, so that the length of the smallest axis $(c)$ is poorly constrained. The fit with a single-body solution is satisfactory for the whole set of visits (Figs. 6 and 12).

The ellipsoid flattening is smaller than the one derived from the photometric analysis $(a / b=2.4, a / c=3.1)$, but it should be taken into account that $c$ is not well constrained. The derived volume would correspond to an effective diameter of $245 \mathrm{~km}$. No IRAS diameter is available for (624) Hektor, but on the other hand the size $416 \times 188 \mathrm{~km}$ found here is consistent with the size estimate $(370 \times 195 \mathrm{~km})$ given by Storrs et al. (1999) from a deconvolution of HST/WFPC data.

Fit residuals indicate that there is no strong evidence of a binary structure. However, to test the binary equal-sized double as hypothesized by Hartmann \& Cruikshank (1978), a fit with a binary model, with either overlapping or separated
Table 4. Comparison to IRAS diameters (unavailable for (624) Hektor). All values are in $\mathrm{km}$.

\begin{tabular}{lcc}
\hline \hline Name & IRAS & Ellipsoid $2 \times R_{\mathrm{v}}$ \\
\hline (15) Eunomia & 255 & 248 \\
(43) Ariadne & 66 & 63 \\
(44) Nysa & 71 & 83 \\
(63) Ausonia & 103 & 87 \\
(216) Kleopatra & 135 & 95 \\
(624) Hektor & - & 245 \\
\hline
\end{tabular}

components, has also been done. The varying parameters are the diameters of the primary and of the secondary, and their separation.

This model does not improve fit residuals in comparison to the single ellipsoid. Due to the geometry of our observations, the data on the FGS $X$-axis are not very sensitive to a binary structure (see Paper I). The best fit is obtained for a "binary" with two overlapping components and with a relatively large diameter ratio (0.9), thus for a shape that, given the resolution of the instrument, is not significantly different from that of a single ellipsoid (see Fig. 16). Thus, our HST/FGS data do not conclusively reject the hypothesis of a dumbbell-shape made of two large and similarly sized bodies. The data $S / N$ ratio together with the limited $(u, v)$ plane coverage are not high enough to separate those two shape models. Nevertheless our analysis suggests that a single-ellipsoid model better matches the data. Observations with the recently installed astrometer FGS\#1, providing higher $S / N$ ratio, would be helpful for a better reconstruction of the shape of (624) Hektor.

\section{Final remarks and perspectives}

Considering that the $S / N$ ratio measured on the averaged S-curves is $>10$ for all the objects, with the exception of (624) Hektor, the technique of data reduction proves to be sufficient to extract relevant shape information from our data. As explained in Paper I, we recall here that, when fit residuals are as low as in the case of (63) Ausonia, the sensitivity of size estimates reach a level of 1-2 mas, at least on favorably projected directions.

The available data allow us to test the binary structure hypothesis, providing valuable information on the asteroid size. For instance the tentative binary models of Cellino et al. (1985), that would be in satisfactory agreement to observed lightcurves, would give too large diameters ratio to be coherent with our HST/FGS data. Also, the inversion procedure applied here, which considered only ellipsoidal figures, provides good indication on the presence of unmodeled shape and/or brightness anomalies, as it can be seen in the cases of (15) Eunomia and (63) Ausonia.

None of the asteroids of this program appears to be a well separated or nearly-contact binary system, with components of similar size, as expected from a binary fission model. Except for (216) Kleopatra, which is best represented as two overlapping ellipsoids (hence resembling a nearly-contact binary structure), the observed asteroids turn out to be, on average, 

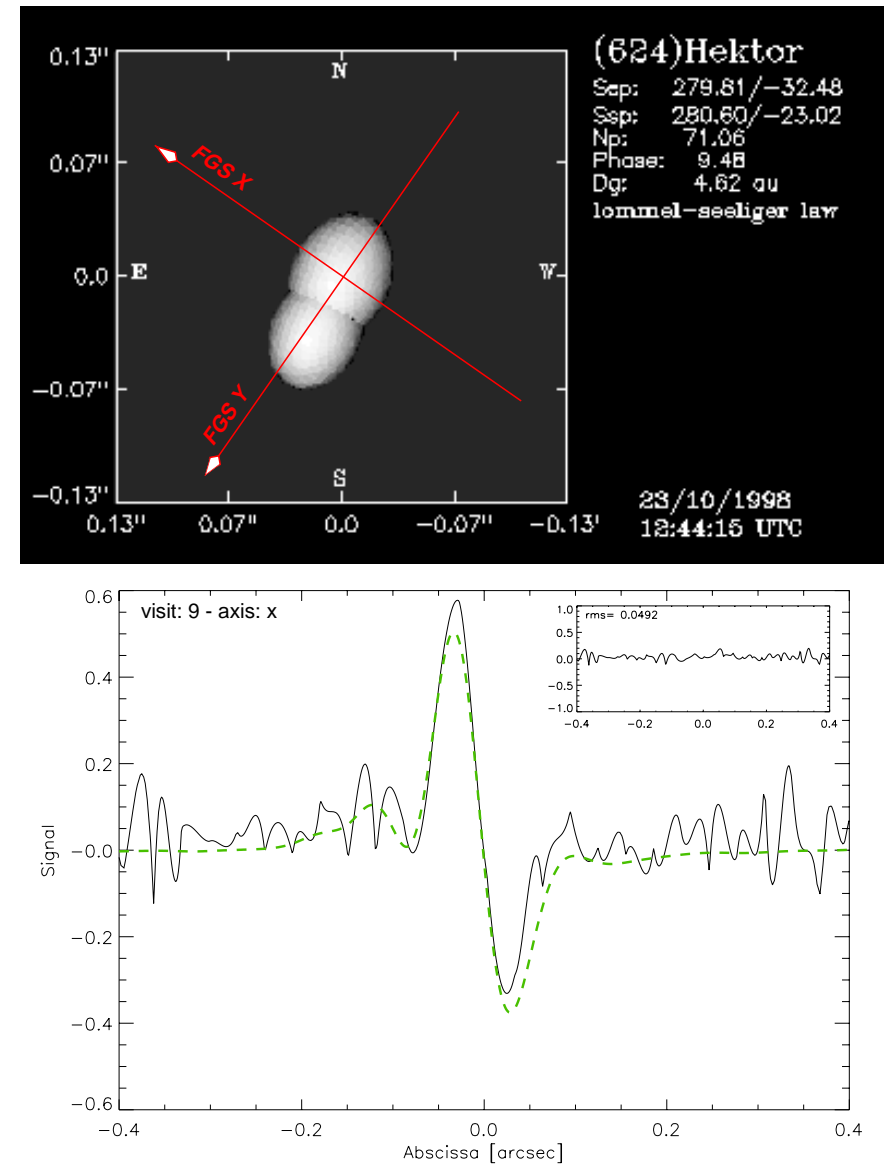

Fig. 16. "Binary" shape model for (624) Hektor on the last visit (top), and interferogram (bottom).

not very different from single triaxial ellipsoids, although some appreciable discrepancies with such a simple shape model are apparent in some cases, as quoted above. For instance, a better fit solution is obtained for (15) Eunomia when considering a single egg-like shaped convex body, while for (43) Ariadne, a contact-binary non-convex shape would provide a better fit to the data. Kaasalainen et al. (2002) give a convex conical shapemodel of (44) Nysa that they suggest to be the signature of a contact structure whose components have different sizes. A more extensive test of such an hypothesis from the HST/FGS data will be done in a forthcoming work.

As for the absolute precision of the size measurements, those derived represent probably lowest boundary values, since we considered uniform brightness distributions. In fact, introducing an a priori limb-darkening would result in slightly larger sizes. Adopting a realistic Minnaert parameter of $k=0.6$ the size is approximately $3 \%$ larger. This would lead to an improved agreement with nominal IRAS results, but in cases, like (216) Kleopatra, in which the resulting shape is very far from a sphere. It is also clear, in any case, that a better determination of the most uncertain axis lengths would be needed to carry out a more detailed comparison with published size data.

In this respect, a whole shape reconstruction at the highest precision reachable by HST/FGS would offer a precious insight on the real shape of these asteroids. Unfortunately, our modelfitting inversion procedure is limited by the short available observing time. This was generally long enough to provide useful indications on the three-dimensional shape of the bodies, but it was nevertheless shorter than $10 \%$ of the rotation period. Moreover, our observations of each object were limited to a single value of the aspect angle. Obviously, more data covering different observing geometries could allow us to retrieve a more complete model of asteroid shapes at the available resolution. In particular, observations made at different aspect (and rotation) angles could be combined to eliminate the limited resolution that is possible in the determination of some of the principal axes.

A further level of investigation will require us also to combine HST data with observations made by other techniques (e.g. radar observations Hudson \& Ostro 1994 or light-curve inversion Kaasalainen \& Torppa 2001). For instance, light-curve amplitudes can help to constrain the surface scattering parameters for a given ellipsoid. Furthermore, photometry can suggest asymmetric shapes that could justify the differences observed between primary and secondary extrema. This refinements represent, in fact, the next step in an analysis that should eventually lead to a reduction process that takes consistently into account, both photometry and HST/FGS interferograms at the same time.

The reduction of the residual uncertainties would allow us to compare the obtained models to the equilibrium figures for fluid bodies (rubble piles) as suggested by Farinella et al. (1981). The available data seem to indicate that the observed asteroids would resemble prolate spheroids (i.e. $b \sim c$ ), which, although not being part of the Jacobi sequence, would suggest low bulk densities. When dealing with rubble pile structures, which are not ideal fluid bodies, however, one should also consider the possible role of internal friction, as suggested by Holsapple (2001), leading to more realistic bulk-densities and macroscopic porosities.

\section{Conclusions}

The direct detection of binary asteroids by long-range observations requires the exploitation of the highest resolutions currently available. For the first time, we have been able to use the FGS astrometer of the HST to study moving targets of the Solar System. The restricted sample of targets was selected mainly on the basis of models derived from photometric observations. Even though no detached binary object was discovered, this criteria has proven to be valuable in selecting targets that present significant shape features.

Due to the rotation of the asteroids around their axis, not only can the sky-projected profile be measured, but it is also possible to infer information about the fully three-dimensional shape, provided that some pole solution can be found to be consistent with FGS data. Moreover, wrong pole solutions can be rejected and nearly-contact binary asteroids revealed. Results are expected to be even better using the upgraded and more sensitive FGSR\#1. Depending on the geometry of the observations, the lengths of the principal axes of the bodies cannot all be determined with the same precision, but observations at different epochs would efficiently overcome such limitations. 
The inversion procedures applied here have proved to be solid, being able to provide best-fit ellipsoidal figures and to test alternative, more complex models. Some evolved binary structures cannot be completely ruled out for some of the objects of our sample based on the available data, although single ellipsoidal shapes are generally a good first approximation of what we have observed. While we hope to obtain new HST/FGS data in the future, we are confident that more detailed analyses of data coming from different sources, including the data presented in this paper, will likely provide stricter constraints to the overall shapes and internal structures of large asteroids and to their past collisional evolution.

Acknowledgements. We are grateful to the FGS team at the STScI, in particular E. Nelan and D.C. Taylor. Important help concerning data reduction and modeling has been provided by D. Loreggia (OATo), F. Gugliemetti (currently at IMPRS, Munich) and J. Berthier (IMCCE). P. Descamps (IMCCE) kindly put at our disposal "MOVIS", the shape modelling and visualization software. D. Hestroffer acknowledges funding from the OATo, and P. Tanga the support of the H. Poincaré fellowship of the Observatoire de la Côte d'Azur.

\section{References}

Cellino, A., Pannunzio, R., Zappalà, V., Farinella, P., \& Paolicchi, P. 1985, A\&A, 144, 355

Cellino, A., Zappala, V., \& Farinella, P. 1989, Icarus, 78, 298

Farinella, P., Paolicchi, P., Tedesco, E. F., \& Zappalà, V. 1981, Icarus, 46,114

Gaffey, M. J., \& Ostro, S. J. 1987, in Lunar and Planetary Institute Conference, $18,310+$

Hartmann, W. K., \& Cruikshank, D. P. 1978, Icarus, 36, 353

Hestroffer, D. 1998, A\&A, 336, 776
Hestroffer, D., Tanga, P., Berthier, J., et al. 2002a, HST/FGS interferometric observations of asteroids, to appear in Mem. Soc. Astron. Italiana

Hestroffer, D., Tanga, P., Cellino, A., et al. 2002b, A\&A, 391, 1123

Hestroffer, D., Berthier, J., Descamps, P., et al. 2002c, A\&A, 392, 729

Hilton, J. L. 1992, in Explanatory supplement to the Astronomical Almanac, 383

Holsapple, K. A. 2001, Icarus, 154, 432

Hudson, R. S., \& Ostro, S. J. 1994, Science, 263, 940

Kaasalainen, M., \& Torppa, J. 2001, Icarus, 153, 24

Kaasalainen, M., Torppa, J., \& Piironen, J. 2002, A\&A, 383, L19

Lattanzi, M. G., Munari, U., Whitelock, P. A., \& Feast, M. W. 1997, ApJ, 485, 328

Leone, G., Paolicchi, P., Farinella, P., \& Zappalà, V. 1984, A\&A, 140, 265

Lupishko, D. F., Akimov, L. A., \& Belskaia, I. N. 1984, in Asteroids, Comets, Meteors, 63

Magnusson, P., Lagerkvist, C., Dahlgren, M., et al. 1994, in Asteroids, Comets, Meteors 1993, IAU Symp., 160, 471

Marchis, F., Hestroffer, D., Cellino, A., Tanga, P., \& Zappalà, V. 1999, IAU Circ., 7308

Minnaert, M. 1941, ApJ, 93, 403

Ostro, S. J., Hudson, R. S., Nolan, M. C., et al. 2000, Science, 288, 836

Parker, J. W., Stern, S. A., Thomas, P. C., et al. 2002, AJ, 123, 549

Seidelmann, P. K., Abalakin, V. K., Bursa, M., et al. 2002, Cel. Mech. Dyn. Astron., 82, 83

Storrs, A., Weiss, B., Zellner, B., et al. 1999, Icarus, 137, 260

Taylor, R. C. in Asteroids, ed. T. Gehrels, 480

Tanga, P., Hestroffer, D., Berthier, J., et al. 2001, Icarus, 153, 451

Weidenschilling, S. J. 1980, Icarus, 44, 807

Zappalà, V., di Martino, M., Scaltriti, F., Djurasevic, G., \& Knezevic, Z. 1983, Icarus, 53, 458

Zappalà, V., \& Knezevic, Z. 1984, Icarus, 59, 436

Zappalà, V., Tanga, P., Cellino, A., di Martino, M., \& Lattanzi, M. G. 1998, HST Program, 7488 